\title{
Artificial Intelligence for Every Individual? It's Easy if You Do it Smart
}

\author{
Srijana Shet \\ Assistant Professor \\ The Yenepoya Institute of Arts, Science, Commerce and \\ Management \\ Yenepoya (Deemed to be University)
}

\author{
Suman Antony Lasrado \\ Assistant Professor \\ St Aloysius College
}

\begin{abstract}
Man-made wit usually referred to as Artificial Intelligence (A.I.) may be a multidisciplinary field whose objective is to robotize exercises that directly need human insight. A.I is dynamical the method we have a tendency to work and live. whether or not you wish to understand what's behind the buzzwords or whether or not you wish to maybe use AI yourself either in an exceedingly personal context or in an exceedingly corporation or different organization. In line with a study by McKinsey International Institute, $\mathrm{AI}$ is calculable to form a further thirteen trillion US bucks useful annually by the year 2030. Despite the fact that $\mathrm{AI}$ is already making tremendous amounts useful to be created in an exceedingly future lies outside the software system trade. In sectors, like retail, travel, transportation, automotive, materials, producing then on. the intense issue zones cared-for in an exceedingly. Here in this paper, I have tried to allow a way of AI technologies and what they will do or cannot do and can also be accustomed to build valuable skills.
\end{abstract}

Keywords - Artificial Intelligence (AI), Artificial Narrow Intelligence (ANI), Artificial General Intelligence (AGI), Artificial Super Intelligence (ASI), Machine Learning (ML), Workflow of data science project, Adversarial attacks.

\section{INTRODUCTION}

In this day and age, innovation is turning extraordinarily fast, and that we square measure connecting with numerous new advances step by step. Here, one amongst the blasting advances of software system engineering is AI that is ready to create another unrest in the world by creating wise machines. Artificial Intelligence is presently encompassing the US. It's straight away operating with Associate in Nursing assortment of subfields, extending from general to express, for instance, self-driving vehicles, enjoying chess, demonstrating hypotheses, enjoying music, Painting, and so on. Artificial intelligence is one of the fascinating and general fields of technology that has an implausible breadth in the future.

Artificial Intelligence consists of 2 words Artificial and Intelligence, wherever Artificial defines "man-made," and intelligence defines "thinking power", thus AI suggests that "an artificial thinking power." So, we are able to outline AI as "It may be a branch of technology that we are able to produce intelligent machines which might behave sort of like a human, suppose like humans, and ready to create choices."[1].

According to the father of Artificial Intelligence, John McCarthy, it is "The science and engineering of making intelligent machines, especially intelligent computer programs".

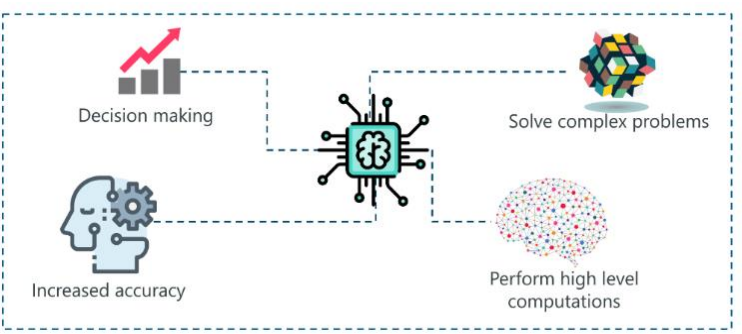

Figure 1 - Artificial Intelligence Courtesy: Edureka

Artificial Intelligence is a method of making a PC, a PC controlled robot, or a product that thinks insightfully, in the comparable way the clever people think. AI is practiced by concentrating on how the human cerebrum thinks, and how people learn, choose, and work while attempting to tackle an issue, and afterward utilizing the results of this investigation as a premise of creating smart programming and frameworks. While exploiting the intensity of the PC frameworks, the interest of humans, led him to ponder, "Can a machine think and carry on as people do?" Along these lines, the advancement of AI began with the goal of making comparative knowledge in machines that we find and respect high in people. [2].

\section{ARTIFICIAL INTELLIGENCE STAGES (AI)}

Artificial intelligence advancements are arranged by their ability to emulate human attributes, the innovation they use to do this, their genuine applications, and the hypothesis of the mind.

AI may be summed up as Perception, Manipulation, Reasoning, Communication, and Learning. Perception is disturbed regarding structure models of the physical world from tactile data (visual, sound, then forth). Manipulation is disturbed regarding articulating extremities (e.g., mechanical arms, motion gadgets) therefore on impact a perfect state within the physical world. The reasoning is disturbed regarding a lot of elevated levels of subjective capacities, for instance, arranging, reaching inferential inferences from a world model, diagnosing, planning, then forth. Communication treats tough comprehension and spending on knowledge of victimization language. Lastly, Learning treats the difficulty of consequently rising framework execution when it is slow and keen about the framework's understanding. 
Nonetheless, despite the fact that computerized reasoning is alluded to as $\mathrm{AI}$ in the media, there are various sorts of AI out there. These three kinds are Artificial Narrow Intelligence (ANI), Artificial General Intelligence (AGI), and Artificial Super Intelligence (ASI).

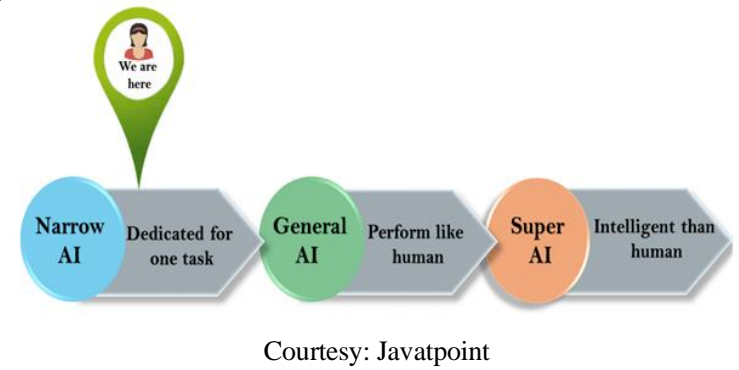

\section{A. ARTIFICIAL NARROW INTELLIGENCE}

Commonly called Weak AI, ANI is the phase of Artificial Intelligence including machines that can perform just a barely characterized set of explicit undertakings. At this stage, the machine doesn't have any reasoning capacity, it just plays out a lot of pre-characterized capacities.

Instances of Weak AI include Siri, Alexa, Self-driving vehicles, Alpha-Go, Sophia the humanoid, etc. Practically all the AI-based frameworks worked till this date falls under the class of Weak AI.

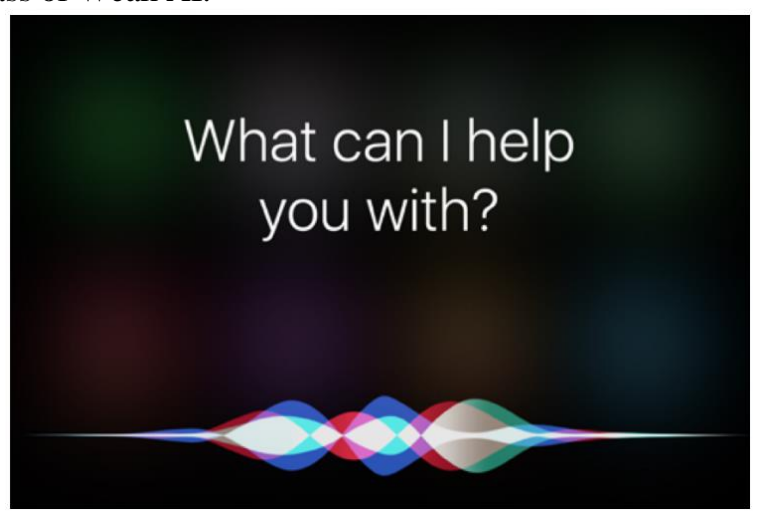

FIGURE 2 - ARTIFICIAL NARROW INTELLIGENCE COURTESY: EDUREKA

\section{B. ARTIFICIAL GENERAL INTELLIGENCE}

Commonly called Strong AI, AGI is the phase in the advancement of Artificial Intelligence wherein machines will have the capacity to think and settle on choices simply like us people. As of now, there are no instances for Strong AI, but it is accepted that we will before long have the option to make machines that are as keen as people.

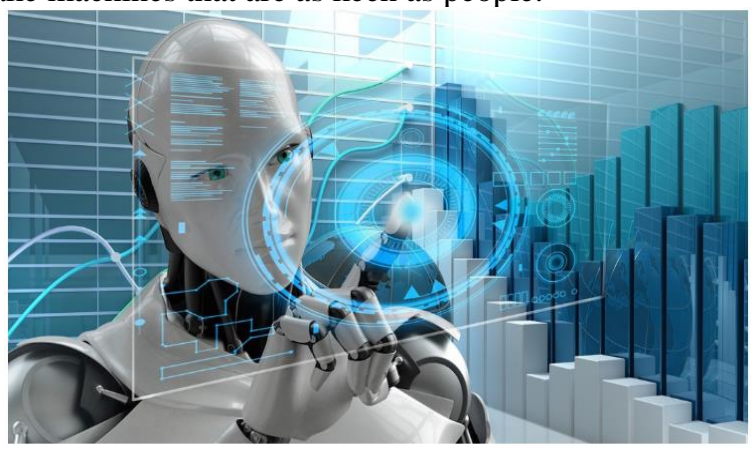

FiguRE 3 - ARTIFICIAL GENERAL INTELLIGENCE

COURTESY: EDUREKA
Strong AI is viewed as a danger to human presence by numerous researchers, including Stephen Hawking who expressed that:

"The development of full artificial intelligence could spell the end of the human race.... It would take off on its own, and re-design itself at an ever-increasing rate. Humans, who are limited by slow biological evolution, couldn't compete and would be superseded."

\section{ARTIFICIAL SUPER INTELLIGENCE}

Artificial Super Intelligence is the phase of Artificial Intelligence when the ability of PCs will outperform people. ASI is as of now a speculative circumstance as portrayed in motion pictures and sci-fi books, where machines have assumed control over the world.

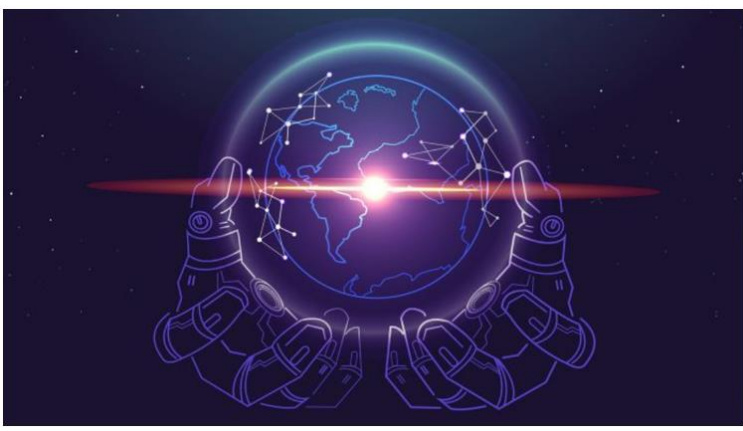

Figure 4 - Artificial Super Intelligence Courtesy: Edureka

I accept that machines are not exceptionally a long way from venturing this stage taking into contemplations our present pace.

"The pace of progress in artificial intelligence (I'm not referring to narrow AI) is incredibly fast. Unless you have direct exposure to groups like Deepmind, you have no idea how fast-it is growing at a pace close to exponential. The risk of something seriously dangerous happening is in the five-year timeframe. 10 years at most." -Elon Musk quotes.

\section{MACHINE LEARNING}

The rise of AI has been generally determined by one apparatus in AI called machine learning. The most generally utilized kind of machine learning is a sort of AI that learns A to $\mathrm{B}$, or contribution to yield mappings. This is called supervised learning. How about we see a few models. On the off chance that the info A is an email and the output B is email spam or not, zero one. At that point this is the center bit of AI used to manufacture a spam channel. Or on the other hand if the information is an audio clip, and the AI's main responsibility is to yield the content transcript, at that point this is speech recognition. More models, on the off chance that you need to enter English and have it yield an alternate language, Chinese, Spanish, something different, at that point this is a machine translation. Or on the other hand the most worthwhile type of supervised learning, of this sort of AI, perhaps be internet promoting, where all the huge online advertisement stages have a bit of AI that inputs some data about an advertisement, and some data about you, and attempts to make sense of, will you click on this 
advertisement or not? By indicating to you the advertisements you're well on the way to tap on, this ends up being exceptionally worthwhile. Perhaps not the most motivating application, yet positively having a huge economic effect today. Or then again on the off chance that you need to assemble a self-driving vehicle, one of the key bits of $\mathrm{AI}$ is in the $\mathrm{AI}$ that takes as information a picture, and some data from their radar, or from different sensors, and yield the situation of different vehicles, so your self-driving vehicle can maintain a strategic distance from different vehicles. Presently, supervised learning has been around for a long time. In any case, it's truly taken off over the most recent couple of years. Why would that be? Suppose on the horizontal axis you plot the measure of information you have for an assignment. Thus, for discourse acknowledgment, this may be the measure of sound information and transcripts you have. In a great deal of enterprises, the measure of information you approach has truly become in the course of the most recent few decades. Because of the rise of the Internet, the rise of PCs. A ton of what used to state bits of paper are presently rather recorded on a computerized PC. Along these lines, we've recently been getting an ever increasing number of information. Presently, suppose on the vertical axis you plot the exhibition of an AI framework. For reasons unknown, on the off chance that you utilize a customary AI framework, at that point the exhibition would develop this way, that as you feed in more information is execution shows signs of improvement. In any case, past a specific point, it didn't beat that. So maybe your discourse acknowledgment framework didn't get substantially more exact, or your internet promoting framework didn't get significantly more precise that is indicating the most important advertisements, even as you show the more information. Computer based intelligence has truly taken off as of late because of the rise of neural systems and profound learning.

\section{WORKFLOW OF DATA SCIENCE PROJECT}

Assume you are beginning another information science venture (which could either be a short examination of one dataset, or a complex multi-year joint effort). In what capacity ought to sort out your work process? Where do you put your information and code? What instruments do you use and why? As a rule, what would it be advisable for you to think about before making a plunge into your information? In the product building industry such inquiries have some ordinarily known answers. Although each product organization may have its one of a kind attributes and characteristics, the center procedures in the vast majority of them depend on the equivalent set up standards, practices and apparatuses.

[4]. For the most part, data students frequently ask us inquiries like "what does a Data Scientist do?". Or then again "what does a day in the information science life resemble?" These inquiries are tricky. The appropriate response can differ by job and company.

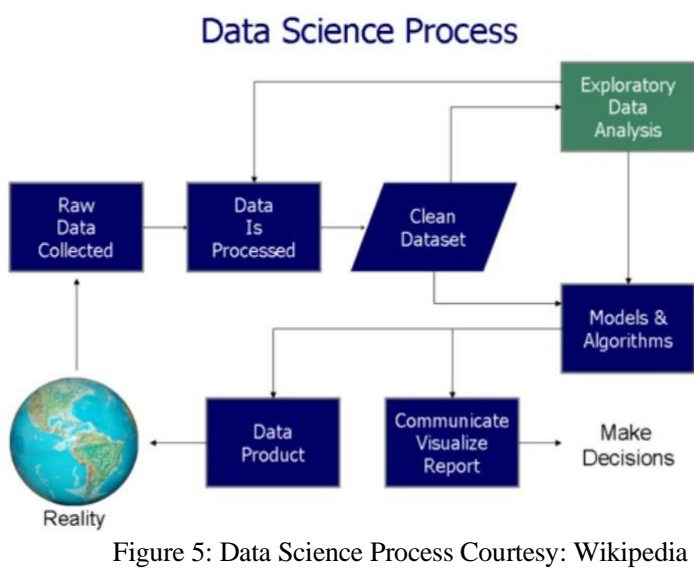

Stage 1: Frame the problem

The primary thing you need to do before you tackle an issue is to characterize precisely what it is. You should have the option to make an interpretation of information inquiries into something noteworthy.

You'll frequently get uncertain contributions from the individuals who have issues. You'll need to build up the instinct to transform scant contributions to noteworthy yields and to pose the inquiries that no one else is inquiring.

Let's assume you're taking care of an issue for the VP Sales of your organization. You should begin by understanding their objectives and the basic why behind their information questions. Before you can begin considering arrangements, you'll need to work with them to obviously characterize the issue. An extraordinary method to do this is to pose the correct inquiries. You should then make sense of what the business procedure resembles, and who the clients are. You need however much setting as could reasonably be expected for your numbers to become experiences.

You ought to pose inquiries like the accompanying: Who are the clients?

For what reason would they say they are purchasing our item?

How would we anticipate if a client is going to purchase our item?

What is not the same as fragments who are performing admirably and those that are performing beneath desires?

What amount of cash will we lose on the off chance that we don't effectively offer the item to these gatherings?

In light of your inquiries, the VP Sales may uncover that they need to comprehend why certain portions of clients have purchased not exactly anticipated. Their ultimate objective may be to decide if to keep on putting resources into these sections, or de-organize them. You'll need to tailor your examination to that issue, and uncover bits of knowledge that can bolster either end.

It's significant that toward the finish of this stage, you have the entirety of the data and setting you have to take care of this issue. 


\section{Stage 2: Collect the raw data required for your problem}

When you've characterized the issue, you'll need information to give you the bits of knowledge expected to turn the issue around with an answer. This piece of the procedure includes thoroughly considering what information you'll need and discovering approaches to get that information, regardless of whether it's questioning inward databases, or buying outer datasets.

You may discover that your organization stores the entirety of their business information in a CRM or a client relationship the board programming platform. You can trade the CRM information in a CSV document for additional examination.

\section{Stage 3: Process the data for analysis}

Since you have the entirety of the crude information, you'll have to process it before you can do any investigation. As a rule, information can be very chaotic, particularly on the off chance that it hasn't been all around kept up. You'll see mistakes that will degenerate your investigation: values set to invalid however they truly are zero, copy esteems, and missing qualities. It's dependent upon you to experience and check your information to ensure you'll get precise bits of knowledge.

You'll need to check for the accompanying basic blunders:

Missing qualities, maybe clients without an underlying contact date

Ruined qualities, for example, invalid sections

Timezone contrasts, maybe your database doesn't consider the diverse timezones of your clients

Date extend mistakes, maybe you'll have dates that has neither rhyme nor reason, for example, information enrolled from before deals began

You'll have to glance through totals of your document lines and sections and test some test esteems to check whether your qualities bode well. On the off chance that you distinguish something that doesn't bode well, you'll have to expel that information or supplant it with a default esteem. You'll have to utilize your instinct here: if a client doesn't have an underlying contact date, does it bode well to state that there was NO underlying contact date? Or on the other hand do you need to chase down the VP Sales and inquire as to whether anyone has information on the client's missing beginning contact dates?

When you're finished working with those inquiries and cleaning your information, you'll be prepared for exploratory data analysis (EDA).

\section{Stage 4: Explore the data}

At the point when your information is perfect, you'll begin playing with it!

The trouble here isn't thinking of thoughts to test, it's coming up with thoughts that are probably going to transform into bits of knowledge. You'll have a fixed cutoff time for your information science venture (your VP Sales is presumably looking out for your examination enthusiastically!), so you'll need to organize your inquiries. '

You'll need to take a gander at the absolute most fascinating examples that can help clarify why deals are decreased for this gathering. You may see that they don't in general will be dynamic via web-based networking media, with not many of them having Twitter or Facebook accounts. You may likewise see that the greater part of them are more established than your general crowd. From that you can start to follow designs you can break down more profoundly.

\section{Stage 5: Perform in-depth analysis}

This progression of the procedure is the place you will need to apply your measurable, scientific and mechanical information and influence the entirety of the information science instruments available to you to crunch the information and discover each understanding you can.

For this situation, you may need to make a prescient model that contrasts your failing to meet expectations gathering and your normal client. You may discover that the age and internet based life action are huge factors in foreseeing who will purchase the item.

On the off chance that you'd posed a ton of the correct inquiries while surrounding your concern, you may understand that the organization has been focusing intensely via web-based networking media advertising endeavors, with informing that is focused on more youthful crowds. You would realize that specific socio economics incline toward being reached by phone instead of by online networking. You start to perceive how the manner in which the item has been promoted is essentially influencing deals: possibly this difficult gathering is certainly not an act of futility! An adjustment in strategies from online networking showcasing to additional in-person associations could make a huge difference to improve things. This is something you'll need to banner to your VP Sales.

You would now be able to consolidate those subjective experiences with information from your quantitative examination to make a story that moves individuals to activity.

\section{Stage 6: Communicate results of the analysis}

It's significant that the VP Sales comprehend why the experiences you've revealed are significant. Eventually, you've been called upon to make an answer all through the information science process. Legitimate correspondence will mean the contrast among activity and inaction on your recommendations.

\section{ADVERSARIAL ATTACKS}

Despite the fact that cutting edge AI is extraordinarily ground-breaking, one of the impediments of current AI innovations, particularly deep learning is that occasionally it tends to be tricked. Specifically, present day AI frameworks are once in a while powerless to adversarial attacks, on the 
off chance that another person sets out purposely to trick your AI framework.

Let's look at an example. Suppose you give an AI framework, this image of a flying creature and request that it characterize it. The AI framework yields this is a hummingbird. Be that as it may, let's make a minor bother to this picture. By minor bother, I intend to change the pixel esteems only somewhat and practically vague change to the vast majority. A similar AI framework at that point says this is rather a sledge. Presently, to an individual you may state, "How is this even conceivable, the image on the correct looks practically indistinguishable from the image on the left?" Truth be told, the progressions are practically impalpable to the natural eye. Yet, an AI framework sees the world uniquely in contrast to you and I do. Along these lines, it is defenseless if an enemy makes changes to an image that could be intangible to you and me yet their outcomes in tricking the AI into intuition this image is something very surprising. We consider this an ill-disposed assault on an AI framework. In PC security, an assault against a protected framework implies an endeavor to cause it to accomplish some different option from what it was proposed to do. Similarly, an ill-disposed assault on an AI framework is an endeavor to cause it to accomplish some different option from what it was proposed to do, for example, attempting to trick it into yielding mistaken groupings. Today, AI is being utilized to filter through hate speech, and attacks like these will reduce the viability of filters.

There are a few attacks that work by changing the physical world too. For instance, a gathering at Carnegie Mellon University had the option to plan a crazy pair of glasses this way. Along these lines, when he keeps an eye on wearing this pair of glasses, he can trick an AI framework into feeling that he is on-screen character Milla Jovovich. I believe it's momentous that simply wearing a couple of glasses like this can trick an AI framework into imagining that this man is a notable on-screen character. An alternate gathering of specialists from UC Berkeley, University of Michigan and different colleges, demonstrated that on the off chance that you influence stickers like these onto a stop sign, you can trick an AI framework into not seeing the stop sign by any stretch of the imagination. It thinks there's something different there other than a stop sign. One fascinating thing about this model is that it would seem that the stop signs simply had some spray painting applied on the head of it. Most people will at present consider this to be a stop sign without any problem. [5].

There is shockingly some measure of misrepresentation on the Internet where individuals are attempting to take cash and installments frameworks or make fake records. The occasions I dealt with hostile to misrepresentation frameworks was one of only a handful hardly any occasions that it truly felt like a lose-lose situation, had a foe, we would set up a safeguard and they would respond. They would dispatch an assault, my groups needed to respond. In some cases, even hours to guard ourselves. Along these lines, I thoroughly consider the following scarcely any years even as AI innovations advances, there will be verticals like that, similar to spam, similar to misrepresentation where groups will be at war. In what my vibe is like a lose-lose situation against enemies. Having said that, I likewise would prefer not to over-publicity the possible harm for adversarial AI frameworks. It is extremely significant for certain applications. Be that as it may, there are likewise a lot of AI applications which are more averse to be liable to ill-disposed assaults. Presently, notwithstanding adversarial attacks, AI tragically can likewise be utilized for some unfavorable or some negative use cases.

\section{CONCLUSION}

AI is a super force, and understanding it permits you to do things that not many individuals on the planet can. AI is at the focal point of another venture to construct computational models of insight. The fundamental supposition that will be that knowledge (human or something else) can be spoken to regarding image structures and representative activities which can be modified in an advanced PC. There is a lot of discussion with respect to whether such a suitably customized PC would be a psyche, or would only mimic one, yet AI scientists need not sit tight for the end to that banter, nor for the speculative PC that could display all of human insight. Parts of astute conduct, for example, taking care of issues, making derivations, learning, and getting language, have just been coded as PC programs, and inside extremely restricted spaces, for example, recognizing sicknesses of soybean plants, AI projects can outflank human specialists. Presently the extraordinary test of AI is to discover methods of speaking to the rational information and experience that empower individuals to do ordinary exercises, for example, holding a wide-extending discussion, or finding their way along a bustling road. Customary advanced PCs might be fit for running such projects, or we may need to grow new machines that can bolster the multifaceted nature of human ideas.

\section{REFERENCES:}

[1] https://www.javatpoint.com/artificial-intelligence-tutorial

[2] https://www.tutorialspoint.com/artificial_intelligence/artificial_int elligence_overview.htm

[3] https://www.edureka.co/blog/types-of-artificial-intelligence/

[4] https://www.kdnuggets.com/2016/03/data-science-process.html

[5] https://www.coursera.org

[6] Goertzel, B. (2007). Artificial general intelligence (Vol. 2). C. Pennachin (Ed.). New York: Springer.

[7] Goertzel, B. (2014). Artificial General Intelligence: Concept, State of the Art, and Future Prospects, Journal of Artificial General Intelligence, 5(1), 1-48

[8] J. Wang, Y. Tang, M. Nguyen and I. Altintas, "A Scalable Data Science Workflow Approach for Big Data Bayesian Network Learning," 2014 IEEE/ACM International Symposium on Big Data Computing, London, 2014, pp. 16-25, doi: 10.1109/BDC.2014.10.

[9] S. W. Berrick, G. Leptoukh, J. D. Farley and Hualan Rui, "Giovanni: A Web Service Workflow-Based Data Visualization and Analysis System," in IEEE Transactions on Geoscience and Remote Sensing, vol. 47, no. 1, pp. 106-113, Jan. 2009, doi 10.1109/TGRS.2008.2003183. 